\title{
Cytogenetics of 16 Chromosome Plants in Pearl Millet
}

\author{
J. V. Pantulu and (Miss) V. Manga \\ Department of Botany, Andhra University, Waltair, India
}

Received December 23, 1970

Trisomics have been the main aneuploid plants used in studies of ge etics and plant breeding of diploid species such as Datura, maize, tomato, spinach, barley etc. (Burnham 1962). Trisomics are especially useful in assigning genes and linkage groups to particular chromosomes. The secondary trisomics, and the $2 n+$ telocentric types may be used to determine which genetic factors are carried in each arm of a particular chromosome. Tertiary trisomics, isochromosomes (secondary trisomics) and telocentrics might some times be the source for the origin of Bchromosomes (Battaglia 1964).

In pearl millet no trisomics were obtained till now and hence could not be used in linkage studies or in other cytogenetic investigations. Earlier, Pantulu (unpublished) had obtained some trisomic plants in the progeny of desynaptic lines. These trisomics, all of them primaries had poor growth habit and were very weak. A few progeny plants obtained from these were all diploids, hence trisomics could not be studied extensively.

The best source of obtaining trisomics is the progeny of triploids (Burnham 1962). In the progeny of a triploid plant of Pennisetum typhoides some trisomics were obtained and the present investigation mainly deals with the meiotic behaviour of two plants with 16 chromosomes.

\section{Materials and methods}

The material for the present study consists of plants with two extra chromosomes, both deficient for short segments in one of the arms. These plants were obtained in the progeny of a cross between a trisomic plant and a diploid. The trisomics employed in the cross were the progeny of a triploid Pennisetum typhoides. For the study of meiosis, young ear-heads were fixed in 3:1 absolute alcohol acetic acid and the customary acetocarmine squash technique was used.

\section{Origin of the plants with two extra chromosomes}

One triploid plant was obtained in the $\mathrm{X}_{2}$ generation of Pennisetum typhoides raised from seed irradiated with gamma rays (Pantulu 1968). Some of the progeny of the triploid, after open pollination, had one extra chromosome in addition to the normal complement of $2 n=14$. The extra chromosome was deficient for a short segment in one of the arms and consequently was acrocentric. The deficient chromosome when entering into a trivalent association, chiasma was found only in the intact arm. 
The chromosome segregation in the plants at anaphase I was irregular as this acrocentric extra chromosome was inducing non-disjunction in the other bivalents of the nucleus.

One plant in the progeny of the triploid was with a translocation involving two non-homologous chromosomes and an extra chromosome. The extra chromosome here also was an acrocentric one. This extra chromosome was not related to the chromosomes involved in translocation.

On selfing the plants with extra chromosomes, plants with two extra chromosomes deficient for short segments in one of their arms were obtained. These plants were double trisomics as the two extra chromosomes entered into two different trivalent associations. These acrocentric extra chromosomes were observed to induce non-disjunction in the other bivalents. In a few cases one or both the trivalents failed to disjoin and moved to one of the poles. These situations led to the irregular distribution at anaphase I. At second division in a small percentage of cells chromatic non-disjunction was observed in the extra chromosomes. Some of these trisomics had 2-4 B-chromosomes.

Plants with extra chromosomes were crossed to normal diploid plants and a progeny of 20 plants was obtained. Two of these twenty plants had two extra chromosomes deficient for short segments in one of their arms. These plants might be the result of chromatid non-disjunction at the second division observed in the PMC's of the plants with one or two extra chromosomes.

\section{Results and conclusions}

These plants with two extra chromosomes were without B-chromosomes. The two extra chromosomes measured about half the length of the shortest chromosomes of the complement and were deficient for short segments in one of their arms and resembled the extra chromosome parent in this character. The extra chromosomes were present invariably in all the PMC's observed. At pachytene stage they were observed to have sub-terminal centromeres and were euchromatic (Fig. 1).

At diakinesis and metaphase I they were pairing only among themselves or left out as univalents but were never observed to pair with the other chromosomes of the complement (Fig. 2). At metaphase I the extra chromosomes were in bivalent association in 86 percent of the cells and in 14 percent of the cells they remained as univalents. The PMC mean chiasma frequency was 12.6 and the mean chiasma frequency varied from 10 to 14 per nucleus giving a total variation of 0.27 . This variation was much higher than in the normal diploid plants but was comparable to the variation observed in plants with 3-4 B-chromosomes of a B-chromosome stock studied (Manga unpublished). The orientation of the extra bivalent at metaphase I was normal and was in line with the other bivalents in the nucleus.

At anaphase I, out of 60 cells examined, in 46 cells the extra bivalent was undergoing regular disjunction which resulted in 8-8 distribution of the chromosomes. In 14 cells this bivalent either failed to disjoin and moved to one of the poles or lagged behind and disjoined later. In a few cases the lagging bivalent was included in one 
of the interphase nuclei without disjoining. Thus these extra chromosomes when forming bivalents were showing chromosomal non-disjunction. These extra chromosomes were inducing non-disjunction in other bivalents at anaphase I. (Fig. 3).
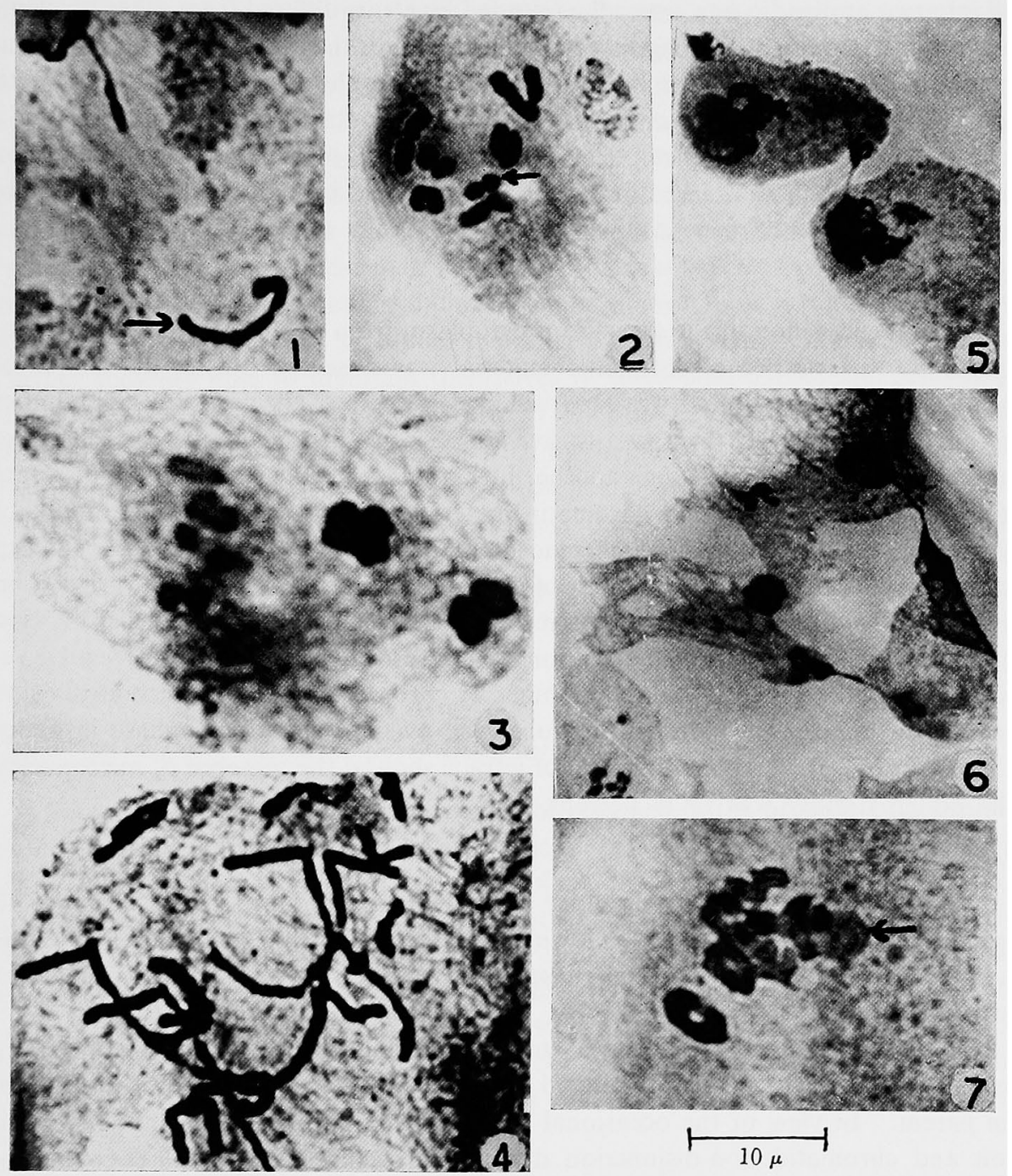

Figs. 1-7. Showing meiosis in a plant with two extra chromosomes. 1, pachytene stage showing the morphology of the two accrocentric extra chromosomes $(\rightarrow)$. 2, diakinesis stage showing three ring and five rod bivalents. One of the rod bivalents being formed by the extra chromosomes $(\rightarrow)$. 3, anaphase I showing non-disjunction of four ring bivalents. 4, showing differentially condensed bivalents and four univalents. 5, showing two cells involved in cytomixis. 6, four cells involved in cytomixis. 7 , metaphase I showing a persistent nucleous $(\rightarrow)$.

These plants with two acrocentric extra chromosomes showed some meiotic irregularities like differential condensation of the chromosomes, cytomixis and persistent nucleoli. 


\section{Differential condensation}

This phenomenon was observed in 27 percent of the cells from diplotene to diakinesis (Fig. 4). The retarded bivalents which were other than the extra bivalent fall into two groups; in one group the retarded bivalents differed from other bivalents in the same nucleus in the degree of condensation, in the second group these bivalents were not only relaxed but feebly stained and were showing hazy outlines. Both the types of bivalents, however, were able to catch up and attain the normal condition of condensation by metaphase I. Similar type of differential condensation of bivalents was also observed in inbred materials and in plants with four or more than four B-chromosomes (Manga unpublished).

\section{Cytomixis}

The phenomenon of the transfer of cytoplasm from one cell to another accompanied by the transfer of chromatic material was observed during the course of meiosis (Fig. 5). Cytomixis was observed in 20 percent of the cells, and the number of cells involved varied from two to five (Fig. 6). Where a number of cells were found to be in contact with one another by cytoplasmic extensions, different stages of transfer of cytoplasm and chromatin material were observed. The amount of cytoplasm and the number of chromosomes involved in cytomixis also showed considerable variation. The amount of chromatin material transferred varied from one bivalent to all the chromosomes in the complement. In all the cells involved in cytomixis the anaphase I distribution was observed to be generally irregular resulting in dyad cells with variable number of chromosomes. The second division was largely regular. Cytomixis was observed even at second telophase in about 0.5 percent of cells. About 4 percent of tetrads showed the presence of micronuclei. The pollen sterility was 19.74 percent.

\section{Persistent nucleoli}

Nucleoli which normally disappar by the end of prophase I were observed to persist even in the other stages following prophase I of meiosis. Out of 200 cells examined at metaphase I and anaphase I, 8 cells were showing a single persistent nucleolus (Fig. 7).

The extra chromosomes appear to be homologous in view of their pairing behaviour and seem to have originated from one of the two extra chromosomes in the parent. In view of the occasional chromosomal non-disjunction, at first division and chromatid non-disjunction during the second division of the deficient chromosomes in the parent any one of the extra chromosomes of the parent can be expected in duplicate in the progeny. The extra chromosome when present singly, pairs with the chromosomes of the normal complement with which it is homologous, but when another exactly similar deficient chromosome is present, there seems to be preferential pairing between the extra chromosomes.

These extra chromosomes represent spare genetic material and hence can be subjected to alterations. These undergo, like B-chromosomes, accumulation and elimination in the populations.

The cytological behaviour of the two extra chromosomes and their effect on 
some nuclear characters are interesting. These extra chromosomes are smaller than the smallest chromosomes of the complement. They possess sub-terminal centromeres and are euchromatic. They never paired with any of the other chromosomes of the complement, but only paired among themselves to form a bivalent or remained as univalents. When forming a bivalent they are exhibiting chromosomal nondisjunction at anaphase I. By their presence they are inducing some abnormal meiotic behaviour such as 1) variation in mean chiasma frequency 2) differential condensation of the other bivalents in the nucleus 3) cytomixis and 4) persistent nucleoli. It may be pointed out here that three of these situations (1,2 and 4) were also observed in plants with 4 or more B-chromosomes (Manga, unpublished). From their behaviour at meiosis in PMC's and their affects it is possible to assume that these extra chromosomes are B-chromosomes in formation. If they accumulate hetero-chromatin they may satisfy all the criteria to treat them as B-chromosomes. Further they are able to maintain themselves in these plants from one generation to other. According to Powell and Burton (1966) the nucleolus organizing activity of the euchromatic accessories (B's) reported by them in the material of $P$. typhoides from Nigeria is of interest as this narrows the gap between the structure and function of the B-chromosomes on the one hand and of the A-chromosomes on the other hand.

The junior author is grateful to the U.G.C. for the award of a Junior Research Fellowship.

\section{Summary}

Plants with two extra chromosomes were obtained in the progeny of a triploid plant. The extra chromosomes were deficient for short segments in one of their arms. Meiotic studies in PMC's of these plants showed that these extra chromosomes were not pairing with any of the other chromosomes of the complement but were only pairing among themselves. In cells with the extra chromosomes there was a significant variation in PMC' chiasma frequency. Meiotic abnormalities like differential condensation of chromosomes, persistent nucleoli and cytomixis were observed. The effect of these extra chromosomes on the behaviour of the other chromosomes was compared with the effect of B's when present in numbers of five or more on the A-chromosomes.

\section{Literature cited}

Battaglia, E. 1964. Cytogenetics of B. chromosomes. Caryologia 17: 245-299.

Charles R. Burnham. 1962. Discussions in Cytogenetics. Burges Publishing Co., Minnesota. Manga, V. 1970. Cytogenetic studies of pearl millet (Pennisetum typhoides Stapf and Hubb). Thesis submitted to the Andhra University, Waltair for the award of the degree of Doctor of Philosophy.

Pantulu, J. V. 1961. Cytological studies in the genus Pennisetum with some cytological observations in the genus Caassia. Ph.D. Thesis, Andhra University, Waltair, India. 
- 1968. Meiosis in an autotriploid pearl millet, Pennisetum typhoides. Carylogia 21: 11-15.

Powell Jerrel, B. and Burton, G. W. 1966. Nucleolar organising accessory chromosomes in pearl millet. Crop Science 6: 131-134. 